Research article

\title{
Information about ADRs explored by pharmacovigilance approaches: a qualitative review of studies on antibiotics, SSRIs and NSAIDs
}

\author{
Lise Aagaard*1,2,3 and Ebba Holme Hansen ${ }^{1,2}$
}

Address: ${ }^{1}$ Department of Pharmacology and Pharmacotherapy, Section for Social Pharmacy, Faculty of Pharmaceutical Sciences, University of Copenhagen, Denmark, ${ }^{2}$ FKL-Research Centre for Quality in Medicine Use, Denmark and ${ }^{3}$ The Danish Medicines Agency, Copenhagen, Denmark

Email: Lise Aagaard* - laa@farma.ku.dk; Ebba Holme Hansen - ehh@farma.ku.dk

* Corresponding author

Published: 3 March 2009

BMC Clinical Pharmacology 2009, 9:4 doi:10.1 186/1472-6904-9-4
Received: 16 July 2008

Accepted: 3 March 2009

This article is available from: http://www.biomedcentral.com/l472-6904/9/4

(c) 2009 Aagaard and Hansen; licensee BioMed Central Ltd.

This is an Open Access article distributed under the terms of the Creative Commons Attribution License (http://creativecommons.org/licenses/by/2.0), which permits unrestricted use, distribution, and reproduction in any medium, provided the original work is properly cited.

\begin{abstract}
Background: Despite surveillance efforts, unexpected and serious adverse drug reactions (ADRs) repeatedly occur after marketing. The aim of this article is to analyse ADRs reported by available ADR signal detection approaches and to explore which information about new and unexpected ADRs these approaches have detected.

Methods: We selected three therapeutic cases for the review: antibiotics for systemic use, non-steroidal anti-inflammatory medicines (NSAID) and selective serotonin re-uptake inhibitors (SSRI). These groups are widely used and represent different therapeutic classes of medicines. The ADR studies were identified through literature search in Medline and Embase. The search was conducted in July 2007. For each therapeutic case, we analysed the time of publication, the strengths of the evidence of safety in the different approaches, reported ADRs and whether the studies have produced new information about ADRs compared to the information available at the time of marketing.

Results: 79 studies were eligible for inclusion in the analysis: 23 antibiotics studies, 35 NSAID studies, 20 SSRI studies. Studies were mainly published from the end of the 1990s and onwards. Although the drugs were launched in different decades, both analytical and observational approaches to ADR studies were similar for all three therapeutic cases: antibiotics, NSAIDs and SSRIs. The studies primarily dealt with analyses of $A D R s$ of the type $A$ and $B$ and to a lesser extent $C$ and $D$, cf. Rawlins' classification system. The therapeutic cases provided similar results with regard to detecting information about new ADRs despite different time periods and organs attacked. Approaches ranging higher in the evidence hierarchy provided information about risks of already known or expected ADRs, while information about new and previously unknown ADRs was only detected by case reports, the lowest ranking approach in the evidence hierarchy.

Conclusion: Although the medicines were launched in different decades, approaches to the ADR studies were similar for all three therapeutic cases: antibiotics, NSAIDs and SSRIs. Both descriptive and analytical designs were applied. Despite the fact that analytical studies rank higher in the evidence hierarchy, only the lower ranking descriptive case reports/spontaneous reports provided information about new and previously undetected ADRs. This review underscores the importance of systems for spontaneous reporting of ADRs. Therefore, spontaneous reporting should be encouraged further and the information in ADR databases should continuously be subjected to systematic analysis.
\end{abstract}




\section{Background}

The thalidomide catastrophe around 1960 and additional experiences such as serious adverse drug reactions to high oestrogen oral contraceptives in the 1960s were probably the main reasons for the increasingly stringent requirements set to document development safety and the establishment of spontaneous reporting systems [1,2]. Over the years, the repeated occurrence of unexpected, serious adverse drug reactions (ADRs) has attracted wide professional and public attention, with the result that doubt has been cast on the effectiveness and quality of drug safety surveillance systems. The COX-2 scandal resulting in worldwide withdrawal of $\operatorname{Vioxx}^{\circledR}$ (rofecoxib) from the market in 2004 is a recent example of an ADR case that emerged unexpectedly and took the world by surprise [3]. Several other ADR cases have been discovered after marketing; well known are fenfluramine and the risk of pulmonal hypertension, vigabatrine and visual field defects and tolcapone and the risk of liver toxicity [4-6]. The repeated occurrence of serious ADR cases after medicines have been released on the market questions the extent to which existing systems and methods for predicting ADRs are effective [7]. Information about the ADR profile of a new medicine appears from observations made during the clinical development process $[8,9]$. The gold standard for the design of these clinical trials is the randomised controlled clinical trial (RCCT) [8,9]. The RCCT was designed to measure efficacy rather than ADRs as outcome. The design of the RCCT as hypothesis testing in itself sets narrow limits for the detection of information about serious and unexpected ADRs due to the short treatment period, the relatively small number of carefully selected participants in the trial, fixed drug doses, and hospital settings that do not reflect the conditions under which the medicines are used after marketing [8,9]. Data on well-recognised, easily detectable ADRs may potentially be observed in RCCTs, but unknown, rare or long-term adverse effects are seldom detected in these trials due to the limitations of the RCCT. Detection of unknown or rare ADRs may include other pharmacovigilance designs, e.g. the spontaneous reporting systems, cohort or case-control studies $[1,10-12]$. This article aims to review ADRs reported by available ADR signal detection approaches and to explore which information about new and unexpected ADRs these approaches have detected.

\section{Methods}

We selected three different therapeutic groups of medicines for review. The groups were characterised by different:

\section{a. Therapeutic groups}

- Antibiotics for systemic use
- Non-steroidal anti-inflammatory drugs (NSAIDs)

- Selective serotonin re-uptake inhibitors (SSRIs)

\section{b. Market launch}

Antibiotics were first marketed in the 1940s and NSAIDs in the 1960s, while SSRIs were not launched until the middle of the 1980s (internal documents, The Danish Medicines Agency).

\section{c. ADR profiles}

The therapeutic categories present different ADR profiles due to their specific pharmacological characteristics and functions.

\section{Literature search}

Studies were identified through Medline (from 1966) and Embase (from 1989) using the following MESH terms: serotonin re-uptake inhibitors, anti-inflammatory agents, non-steroidal, anti-bacterial agents, adverse drug reaction reporting systems, pharmacoepidemiology and the key words: adverse drug reactions and information in combination. The literature search was conducted in July 2007 without language restriction. Studies written in non-European languages were later excluded. To be considered relevant for this review, articles had to be empirical in origin and focus on signal detection. Titles and abstracts of the search results were screened and relevant articles identified. The reference lists of included publications were hand-searched for possible additional relevant studies. Non peer-reviewed articles or unpublished observations were not considered. A flow chart of the study selection process for the therapeutic cases is illustrated in figure 1.

\section{Characteristics of the included studies}

We developed a taxonomy inspired by general guidelines for pharmacoepidemiological research to analyse the studies systematically [13]. The taxonomy covers the following characteristics: publication year, design, method, explored medicine and adverse drug reactions, geographic setting, sampling period, sample size, outcome measures and results. We extracted and compared the results of published empirical studies in which various signal detection methods were used. Extracted information was entered into data sheets, one for each article. Data were extracted and handled by the first author and checked by the second author.

\section{Analyses}

For each of the three selected therapeutic groups, we analysed the time of publication, the strengths of the evidence in the different approaches, reported ADRs and whether the studies had produced new information about ADRs 


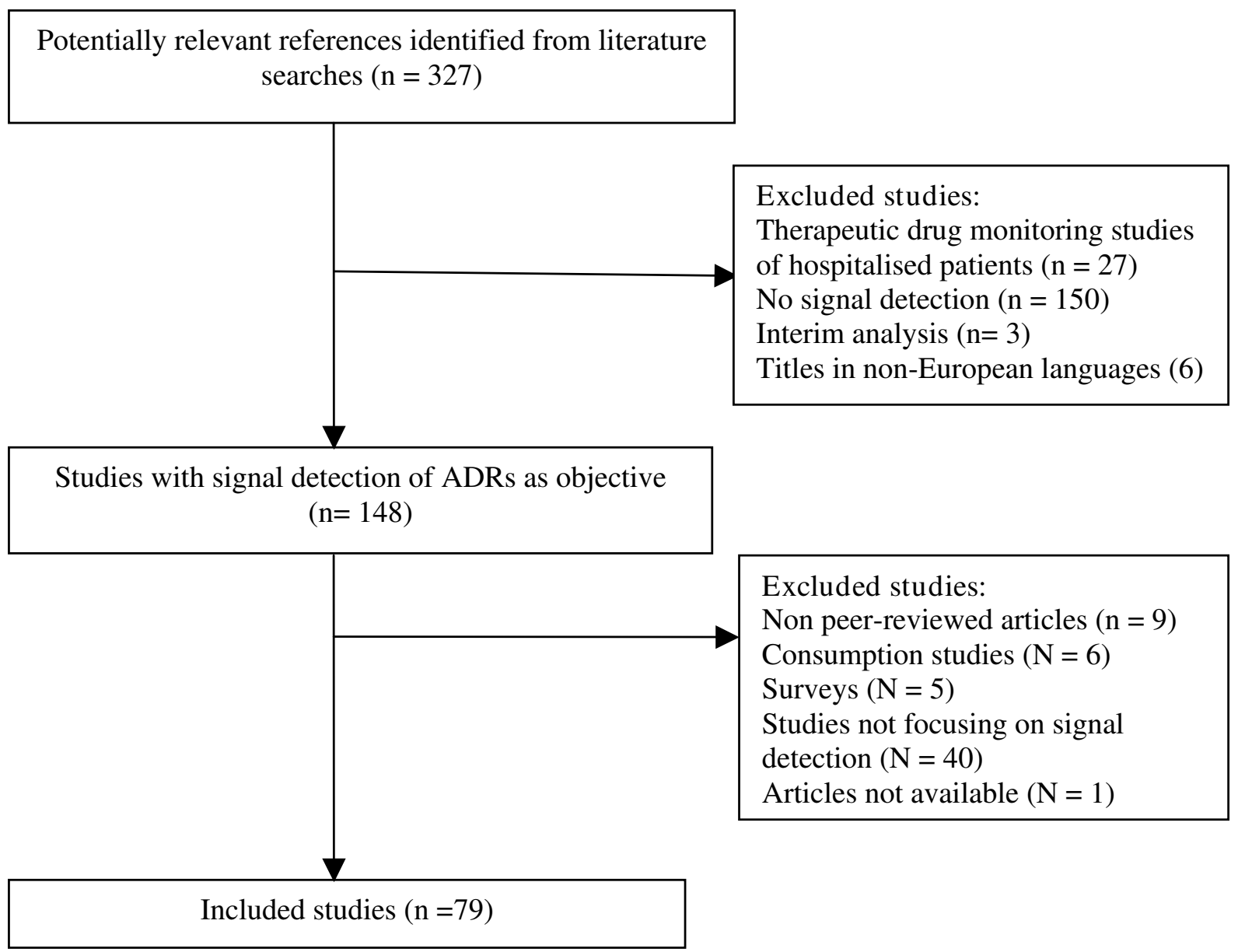

\section{Figure I}

Flow chart of the study selection process for the cases.

compared to the information available at the time of marketing.

\section{Classification of the tested/detected ADRs}

For each included literature reference, the ADRs tested or detected via the various signal detection approaches were classified according to Rawlins' classification system [14]. An overview of the classification system is shown in table 1. The reported/detected ADRs were also classified according to System Organ Classes in keeping with MedDRA terminology [13].

\section{Classification of applied approaches}

The explored approaches were classified into analytical or observational approaches according to Strom's definitions [13]. Case-control and cohort studies are classified as analytical methods, while spontaneous reporting, case series/case reports and PEM studies are observational [13].

Time of publication

For each therapeutic group, we analysed whether there was a connection between time of publication and the applied study design.

\section{Strength of evidence}

Evidence-based medicine operates with an evidence hierarchy for evaluating the quality of the various study designs used for therapeutic studies [13]. At the top of this hierarchy are the meta-analyses (level 1), followed by RCCTs at the second level and other controlled trials at the third level. Cohort studies are placed at the fourth level, followed by case-control studies at the fifth level. At the 
Table I: Rawlins' classification system of ADRs

\begin{tabular}{|c|c|}
\hline Type & Definition \\
\hline \multirow[t]{4}{*}{ A } & Dose-dependent ADRs related to the pharmacological effect of the drug: \\
\hline & - Increased pharmacological effect \\
\hline & - ADRs that occur secondarily to the desired pharmacological effect \\
\hline & - ADRs due to other well known pharmacological effects \\
\hline \multirow[t]{3}{*}{ B } & Sensitivity reactions - not dose-dependent \\
\hline & - Allergic reactions \\
\hline & - Idiosyncratic reaction \\
\hline \multirow[t]{4}{*}{$\mathrm{C}$} & Long-term ADRs \\
\hline & - Carcinogines \\
\hline & - Teratogenes \\
\hline & - Chronic organ damage \\
\hline \multirow[t]{4}{*}{$\mathrm{D}$} & Drug-drug interactions \\
\hline & - Pharmacodynamic \\
\hline & - Pharmacokinetic \\
\hline & - Non-classifiable \\
\hline
\end{tabular}

bottom of the evidence hierarchy are cross-sectional surveys (level 6) and anecdotal case reports (level 7) [13].

\section{Results}

The literature search identified 327 potentially relevant references for all three therapeutic groups, 149 of which were selected from the titles and abstracts and further screened for relevance. Eventually 79 references were included in this analysis. A flow chart of the selection and exclusion process is illustrated in figure 1 . The included studies were distributed on the three therapeutic cases as follows: antibiotics: 23 studies; NSAID: 35 studies; SSRI: 20 studies. One reference was not accessible.

\section{ADR detection approaches applied}

Table 2 provides an overview of the categorisation of the designs used in the included studies and their rank in the evidence hierarchy [13]. As the table indicates, the majority of the included studies dealt with analyses of data reported in Prescription Event Monitoring (PEM) programs and ADRs reported to national ADR databases, approaches ranking at levels six and seven in the evidence hierarchy.

\section{Study characteristics}

Tables 3, 4 and 5 display the characteristics and descriptions of the analysed studies for each therapeutic case [15-

Table 2: The analysed studies categorised by study design

\begin{tabular}{|c|c|c|c|c|c|}
\hline Study design & Rank in evidence hierarchy & & $\begin{array}{l}\text { Therapeutic } \\
\text { cases }\end{array}$ & & \\
\hline & & Antibiotics & NSAIDs & SSRIs & Total \\
\hline Cohort & 4 & 5 & 4 & 1 & 10 \\
\hline Case control & 5 & 2 & 3 & 2 & 8 \\
\hline PEM* & 6 & 2 & 9 & 4 & 15 \\
\hline National ADR databases & 7 & 7 & 14 & 11 & 32 \\
\hline Case series & 7 & 2 & 3 & 1 & 6 \\
\hline Case reports & 7 & 5 & 2 & 1 & 8 \\
\hline Total number of studies & & 23 & 36 & 20 & 79 \\
\hline
\end{tabular}

*Prescription Event Monitoring Studies 
Table 3: Characteristics of studies of the occurrence of ADRs related to antibiotics use

\begin{tabular}{|c|c|c|c|c|c|c|c|c|}
\hline Reference & Setting & Medicines & ADRs & $\begin{array}{l}\text { Sampling } \\
\text { period }\end{array}$ & Sample size & $\begin{array}{l}\text { Outcome } \\
\text { measures }\end{array}$ & $\begin{array}{l}\text { Results } \\
(95 \% \mathrm{CI})\end{array}$ & $\begin{array}{l}\text { Type of } \\
\text { ADRs }\end{array}$ \\
\hline \multicolumn{9}{|l|}{ Case control studies } \\
\hline Czeizel 1999 [15] & $\mathrm{HU}$ & Erythromycin & Teratology & $1980-1996$ & $\begin{array}{l}\text { II } 3 \text { cases/38, I5I } \\
\text { controls }\end{array}$ & OR & I.I; $0.5-2.3$ & $\mathrm{C}$ \\
\hline Seeger 2006 [16] & & Fluoroquinolones & $\begin{array}{l}\text { Achilles tendon } \\
\text { rupture }\end{array}$ & $|997-200|$ & $\begin{array}{l}947 \text { cases/ } \\
18,940 \text { controls }\end{array}$ & OR & $1.2 ; 0.9-1.7$ & B \\
\hline \multicolumn{9}{|l|}{ Cohort studies } \\
\hline Chysky 199I[17] & $\mathrm{DE}$ & Ciprofloxacin & Not specified & 44 days & 634 patients & $\%$ ADRs & $\begin{array}{l}\text { Different } \\
\text { categories } \\
\text { reported }\end{array}$ & $A / B$ \\
\hline Derby 1993 [18] & $\mathrm{AU}$ & Flucloxacillin & $\begin{array}{l}\text { Cholestatic } \\
\text { hepatitis }\end{array}$ & 45 days & 132,087 patients & PRR/I00,000 users & $7.6 ; 3.5-13.9$ & B \\
\hline Jick 1994 [19] & $\mathrm{AU}$ & Flucloxacillin & $\begin{array}{l}\text { Cholestatic } \\
\text { hepatitis }\end{array}$ & $1991-1992$ & 77,552 patients & $\begin{array}{l}\text { PRR/ } \\
100,000 \text { users }\end{array}$ & $6.5 ; 2.7-15.1$ & B \\
\hline Derby 1993b [20] & $\mathrm{AU}$ & Erythromycin & $\begin{array}{l}\text { Cholestatic } \\
\text { hepatitis }\end{array}$ & - & 366,064 patients & PRR/I00,000 users & 3,$6 ; 1.9-6.1$ & B \\
\hline Heymann 2007 [2I] & Israel & Penicillins & Pemphigus & $|997-200|$ & 150,000 patients & OR & $2.03 ; 1.56-2.64$ & B \\
\hline \multicolumn{9}{|l|}{ PEM } \\
\hline Clark 200I [22] & UK & Fluoroquinolones & $\begin{array}{l}\text { Cardiovascular } \\
\text { events }\end{array}$ & $|988-199|$ & 36,410 patients & $\begin{array}{l}\text { CRR } \\
\text { (crude relative } \\
\text { risk) }\end{array}$ & $\begin{array}{l}\text { Atrial fibrillation: } \\
\text { I.0; } 0.02-8.92\end{array}$ & B \\
\hline Inman I994 [23] & UK & Fluconazole & All & $1988-1989$ & 15,015 patients & Frequencies & $\begin{array}{l}\text { Different } \\
\text { categories } \\
\text { reported }\end{array}$ & A \\
\hline \multicolumn{9}{|l|}{$\begin{array}{l}\text { National ADR } \\
\text { databases }\end{array}$} \\
\hline Polimeni 2006 [49] & Sicilian & Antibacterials & All & 1998-2002 & I585 cases & ADRs & $\begin{array}{l}\text { Different } \\
\text { categories } \\
\text { reported }\end{array}$ & A \\
\hline Sachs 2006 [24] & $\mathrm{DE}$ & Fluoroquinolones & Anaphylaxis & $1993-2004$ & 204 cases & PRR $>2$ & $\begin{array}{l}\text { Moxifloxacin: } 2.1 \text {; } \\
\text { Ofloxacin: } 2.3 \\
\text { Ciprofloxacin: } 2.3 \\
\text { Levofloxacin: } 2.0\end{array}$ & B \\
\hline Fleisch 2000 [25] & $\mathrm{CH}$ & Levofloxacin & Tendinopathy & $1986-1999$ & $\begin{array}{l}19 \text { cases } / 460 \\
\text { non-cases }\end{array}$ & Reporting rate & $\begin{array}{l}\text { Different } \\
\text { categories } \\
\text { reported }\end{array}$ & B \\
\hline Leone 2003 [26] & IT & Fluroquinolones & Not specified & $|999-200|$ & $\begin{array}{l}432 \text { cases/ } \\
10,011 \text { non cases }\end{array}$ & Reporting rate & $\begin{array}{l}\text { Different } \\
\text { categories } \\
\text { reported }\end{array}$ & A \\
\hline Pierfitte 2000 [27] & $\mathrm{FR}$ & Sparfloxacin & Phototoxicity & $1994-1996$ & 371 cases & RtR/I000 patients & 0.4 & B \\
\hline Frothingham 2005 [28] & US & Gatifloxacin & $\begin{array}{l}\text { Glucose } \\
\text { homeostatis } \\
\text { abnormalities }\end{array}$ & $1997-2003$ & $\begin{array}{l}453 \text { cases/ } \\
\text { I } 427 \text { non cases }\end{array}$ & $\begin{array}{l}\text { Reporting rate// } 0^{7} \\
\text { prescriptions }\end{array}$ & 477 & $A$ \\
\hline Hedenmalm 1996 [29] & SE & Fluorquinolones & $\begin{array}{l}\text { Sensory } \\
\text { disturbances }\end{array}$ & $1965-1993$ & 37 cases & ADRs & $\begin{array}{l}\text { Different } \\
\text { categories } \\
\text { reported }\end{array}$ & A \\
\hline
\end{tabular}


Table 3: Characteristics of studies of the occurrence of ADRs related to antibiotics use (Continued)

\begin{tabular}{|c|c|c|c|c|c|c|c|c|}
\hline Case series & & & & & & & & \\
\hline Abouesh 2002 [30] & - & $\begin{array}{l}\text { Fluorquinolones } \\
\text { Macrolides }\end{array}$ & Mania & - & 102 cases & Case review & Case review & B \\
\hline Smith 2005 [3I] & - & $\begin{array}{l}\text { Doxycycline } \\
\text { Minocycline }\end{array}$ & ADRs & $1966-2003$ & 130 cases & Incidences & $\begin{array}{l}\text { Doxycycline: } \\
\text { 0-61\% } \\
\text { Minocycline: } \\
\text { II.7-83.3\% }\end{array}$ & A \\
\hline \multicolumn{9}{|l|}{ Case reports } \\
\hline Hällgren 2003 [32] & - & Ciprofloxacin & $\begin{array}{l}\text { Steven-Johnson } \\
\text { syndrome }\end{array}$ & $1988-2000$ & 8 cases & $\begin{array}{l}\text { IC pr. } 100,000 \\
\text { patients }\end{array}$ & 0.045 & B \\
\hline Warner 2000 [33] & - & Clarithromycin & $\begin{array}{l}\text { Acute Psychotic } \\
\text { Stress }\end{array}$ & - & I case & $\begin{array}{l}\text { Causality } \\
\text { assessment }\end{array}$ & Possible & A \\
\hline ADRAC 1992 [34] & - & Flucloxacillin & $\begin{array}{l}\text { Cholestatic } \\
\text { hepatitis }\end{array}$ & - & I case & Case review & Case review & B \\
\hline Greco 1997 [35] & - & Clarithromycin & $\begin{array}{l}\text { Glossitis, } \\
\text { stomatitis, black } \\
\text { tongue }\end{array}$ & - & I case & Case review & Case review & $B$ \\
\hline Björnsson 1996 [36] & - & Doxycycline & Liver reactions & $1966-1995$ & 23 cases & $\begin{array}{l}\text { Causality } \\
\text { assessment }\end{array}$ & $\begin{array}{l}\text { Likely }(n=3) \\
\text { Possible }(n=8)\end{array}$ & B \\
\hline
\end{tabular}

92]. The tables show that the studies primarily dealt with analyses of ADRs of the type A and $\mathrm{B}$, and to a lesser extent $\mathrm{C}$ and $\mathrm{D}$. The evidence level of ADRs varied widely; some of the ADRs were documented in both the analytical and observational studies, others in only one of the designs.

\section{Data sources}

Case-control studies were carried out on data from various national registers and/or data from spontaneous ADR databases, physicians' databases such as the General Practitioners' database in the UK and Health Insurance Databases $[15,16,37,38,72,73]$. The studies were reported in the literature from the mid-1980s to the end of the 1990s. Cohort studies analysed ADR data collected from the mid1980 s to the end of the 1990s. The cohort studies varied in size from less than 20,000 patients to between 20,00050,000 and more than 100,000 patients $[17,19,21,40,41]$. The PEM studies were conducted in the UK at the Drug Safety Unit in Southampton, and were based on data collected from the mid-1980s to the end of the 1990s [22,23,58-66,86-89]. Studies analysing spontaneously reported ADRs were conducted on large spontaneous reporting databases such as the French, American, British and the Uppsala Monitoring Centre WHO database [44$48,51,55-57,62,74-76,79,82,83,85]$.

\section{Design and historical perspective}

The antibiotic studies were published from 1990 and onwards, most of them from 1995. Cohort studies were published during 1990-1994, while the PEM studies, spontaneous reporting, case reports/case series primarily were published after 1995 . The majority of the NSAID studies were published after year 2000. The SSRI studies were published from 1990 to present, most of them from 1995 to 2005. Table 6 shows the distribution of the analysed studies by type of approach, therapeutic case, and time of publication. For all therapeutic cases, data were collected and the studies published a long time after the drugs were first marketed. Despite the decades of difference in market launches for the therapeutic cases, the studies are mainly published from the end of the 1990s and on. Data were collected earlier.

\section{Explored and detected ADRs}

\section{Antibiotics}

ADRs from newer types of antibiotics, such as fluoroquinolones, have been reported much more frequently in the literature than ADRs from the older antibiotics, such as penicillins and macrolides [15-17,19,20,22$24,26,29,33,34,36]$. The studies explore a possible risk between the use of antibiotics and the risk of liver, cardiovascular, CNS and dermatological ADRs [18$20,22,24,27,29,30,32,33,36]$. Three cohort studies documented a correlation between cholestatic hepatitis and the use of flucloxacillin [18-20]. Increased risk of palpitation from the use of norfloxacin compared to ciprofloxacin/ofloxacin was demonstrated [22]. Cohort studies further demonstrated a risk of pemphigus related to penicillins, liver injury related to flucloxacillin and erythromycin [18-21]. CNS and dermatological ADRs from treatment with antibiotics have been reported rarely and on the case report level $[30,32,33]$. New information about ADRs was only produced by case reports: acute psychotic stress and glossitis/black tongue [34,35]. 
Table 4: Characteristics of studies of the occurrence of ADRs related to NSAID use

\begin{tabular}{|c|c|c|c|c|c|c|c|c|}
\hline Reference & Setting & Medicines & ADRs & $\begin{array}{l}\text { Sampling } \\
\text { period }\end{array}$ & Sample size & $\begin{array}{l}\text { Outcome } \\
\text { measures }\end{array}$ & $\begin{array}{l}\text { Results } \\
\text { (95\%Cl) }\end{array}$ & $\begin{array}{l}\text { Type of } \\
\text { ADRs }\end{array}$ \\
\hline \multicolumn{9}{|l|}{$\begin{array}{l}\text { Case control } \\
\text { studies }\end{array}$} \\
\hline Hernandez-Diaz 200। [37] & UK & NSAIDs & $\begin{array}{l}\text { Gastrointestinal } \\
\text { events }\end{array}$ & $1993-1998$ & $\begin{array}{l}2,105 \text { cases/ } \\
\text { II,500 controls }\end{array}$ & OR & $1.8 ; 1.3-2.4$ & $A$ \\
\hline Mockenhaupt 2003 [38] & DE/US & NSAIDs & $\begin{array}{l}\text { Steven-Johnson } \\
\text { syndrome }\end{array}$ & $1989-1995$ & $\begin{array}{l}245 \text { cases/ } \\
\text { I } 147 \text { controls }\end{array}$ & PRR & 34,$95 ; 11-105$ & B \\
\hline Lacroix 2004 [39] & FR & NSAIDs & Liver injury & $1998-2000$ & $\begin{array}{l}88 \text { cases/ } \\
178 \text { controls }\end{array}$ & OR & $\begin{array}{l}\text { Women: } \\
6.49 ; 1.67-25.16 \\
\text { Men: I.06; 0.36-3.12 }\end{array}$ & B \\
\hline \multicolumn{9}{|l|}{ Cohort studies } \\
\hline Lipworth 2004 [40] & DK & Ibuprofen & Mortality & $1989-1995$ & $\begin{array}{l}113,538 \\
\text { patients }\end{array}$ & $\begin{array}{l}\text { SMR } \\
\text { (standard mortality } \\
\text { rate) }\end{array}$ & $1.21 ; 1.19-1.24$ & $\mathrm{~A} / \mathrm{B}$ \\
\hline Ashworth 2004 [4I] & $\mathrm{CA}$ & $\begin{array}{l}\text { Diclofenac } \\
\text { Naproxen } \\
\text { Arthrotec }\end{array}$ & Mortality & $1991-1994$ & 18,424 patients & OR & $\begin{array}{l}\text { Arthrotec: } 1.4 ; 0.9-2.1 \text {. } \\
\text { Diclofenac: } 2.0 ; 1.3-3.1 . \\
\text { Naproxen: } 3.0 ; 1.9-4.6\end{array}$ & $A / B$ \\
\hline Morant 2004 [42] & UK & NSAIDs & $\begin{array}{l}\text { Gastrointestinal } \\
\text { haemorrhage }\end{array}$ & $|987-200|$ & $\begin{array}{l}628000 \text { patient } \\
\text { year }\end{array}$ & PRR & $0.84 ; 0.60-1.17$ & A \\
\hline Martin 2000 [43] & UK & Meloxicam & $\begin{array}{l}\text { Gastrointestinal } \\
\text { events }\end{array}$ & $1996-1997$ & 19,087 patients & $\begin{array}{l}\text { Events/ } \\
1000 \text { patient- } \\
\text { months of } \\
\text { exposure }\end{array}$ & $\begin{array}{l}\text { Dyspepsia: } 28.3 \\
\text { Gastrointestinal } \\
\text { haemorrhage: } 0.4\end{array}$ & $A+B$ \\
\hline \multicolumn{9}{|l|}{ National ADR databases } \\
\hline Lugardon 2004 [44] & FR & $\begin{array}{l}\text { COX-2 } \\
\text { inhibitors }\end{array}$ & $\begin{array}{l}\text { Oeso-gastro- } \\
\text { duodenal events: }\end{array}$ & $2000-2002$ & $\begin{array}{l}505 \text { cases/ } \\
2,525 \text { non-cases }\end{array}$ & OR & $14.9 ; 9.3-23.7$ & $A$ \\
\hline Durrieu 2005 [45] & FR & $\begin{array}{l}\text { COX-2 } \\
\text { inhibitors }\end{array}$ & $\begin{array}{l}\text { Arterial } \\
\text { hypertension }\end{array}$ & $2000-2003$ & 34 cases & OR & $3.3 ; 1.6-6.9$ & $A$ \\
\hline Clinard 2004 [46] & FR & NSAIDs & $\begin{array}{l}\text { Excess risk of } \\
\text { adverse drug } \\
\text { reactions }\end{array}$ & $1995-1999$ & $\begin{array}{l}3983 \text { cases/ } \\
54,583 \text { non- cases }\end{array}$ & OR & $\begin{array}{l}\text { Different categories } \\
\text { reported }\end{array}$ & $B$ \\
\hline Brinker 2004 [47] & US & $\begin{array}{l}\text { COX-2 } \\
\text { inhibitors }\end{array}$ & Hypertension & $<2002$ & 34 cases & $\begin{array}{l}\text { Reporting rate/ } \\
10^{6} \text { person years }\end{array}$ & $\begin{array}{l}\text { Rofecoxib: } 5.0 \\
\text { Celecoxib: } 1.3\end{array}$ & A \\
\hline La Grenade 2005 [48] & US & $\begin{array}{l}\text { cOX-2 } \\
\text { inhibitors } \\
\text { Meloxicam }\end{array}$ & $\begin{array}{l}\text { Steven-Johnson } \\
\text { syndrome } \\
\text { Toxic Epidermal } \\
\text { Necrolysis }\end{array}$ & $<2004$ & 123 cases & $\begin{array}{l}\text { Reporting rate/ } \\
\text { 106person years }\end{array}$ & $\begin{array}{l}\text { Valdecoxib: } 49 \\
\text { Celecoxib: } 6 \\
\text { Rofecoxib: } 3\end{array}$ & B \\
\hline Polimeni 2006 [49] & Sicilian & NSAIDs & All & 1998-2002 & I585 cases & PRR & $\begin{array}{l}\text { Hepatitis: } 14.20 \\
\text { Vasculitis: } 7.72 \\
\text { Hypertension: } 15.40\end{array}$ & B \\
\hline Conforti 200I [50] & IT & NSAIDs & $\begin{array}{l}\text { Gastrointestinal } \\
\text { events }\end{array}$ & $1996-1999$ & $\begin{array}{l}705 \text { cases/ } \\
10,608 \text { non cases }\end{array}$ & $\%$ ADRs & $\begin{array}{l}\text { Nimesulid: } 10.4 \\
\text { Diclofenac: } 21.2 \\
\text { Ketoprofen: } 1.7 \\
\text { Piroxicam: } 18.6\end{array}$ & $A$ \\
\hline Ahmad $2002[5 \mathrm{I}]$ & US & $\begin{array}{l}\text { COX-2 } \\
\text { inhibitors }\end{array}$ & Renal failure & $1969-2000$ & $\begin{array}{l}\text { Celecoxib: } 122 \\
\text { cases } \\
\text { Rofecoxib: } 142 \\
\text { cases }\end{array}$ & Case review & Case review & $A$ \\
\hline Puijenbroek 2000 [52] & NL & $\begin{array}{l}\text { NSAIDs } \\
\text { Diuretics }\end{array}$ & Drug interactions & $1990-1999$ & $\begin{array}{l}305 \text { cases } / \\
9517 \text { non cases }\end{array}$ & OR & OR: $2.0,1.1-3.7$ & $D$ \\
\hline Lapeyre-Mestre 2004 [53] & FR/ES & NSAIDs & Hepatic events & $|982-200|$ & 29,486 cases & OR & $\begin{array}{l}\text { Different OR calculated } \\
\text { for NSAIDs. }\end{array}$ & B \\
\hline
\end{tabular}


Table 4: Characteristics of studies of the occurrence of ADRs related to NSAID use (Continued)

\begin{tabular}{|c|c|c|c|c|c|c|c|c|}
\hline Leone 1999 [54] & IT & Nimesulide & Renal impairment & $1988-1997$ & $\begin{array}{l}\text { IIcases/ } \\
7438 \text { non cases }\end{array}$ & $\begin{array}{l}\text { Causality } \\
\text { assessment }\end{array}$ & $\begin{array}{l}\text { Possible }(n=6) \\
\text { Probable }(n=4) \\
\text { Certain }(n=1)\end{array}$ & $\mathrm{A}$ \\
\hline Brown 1998 [55] & UK & $\begin{array}{l}\text { Tiaprofenic } \\
\text { acid }\end{array}$ & Cystitis & $1981-1996$ & $\begin{array}{l}221 \text { cases/ } \\
1327 \text { non cases }\end{array}$ & $\begin{array}{l}\text { ADRs } / 10^{5} \\
\text { prescriptions }\end{array}$ & $\begin{array}{l}\text { 1991: } 4.2 \\
\text { 1992: } 5.9 \\
\text { 1993: } 4.2 \\
\text { 1994: } 34.4 \\
\text { 1995: } 18.5 \\
\text { 1996: } 6.5\end{array}$ & B \\
\hline Verrico 2003 [56] & US & $\begin{array}{l}\text { COX2- } \\
\text { inhibitors }\end{array}$ & Not specified & 1999-2002 & 24 cases & $\begin{array}{l}\text { Causality } \\
\text { assessment }\end{array}$ & $\begin{array}{l}\text { Possible }(n=29) \\
\text { Probable }(n=16)\end{array}$ & A \\
\hline Kahn I997 [57] & US & NSAIDs & $\begin{array}{l}\text { Necrotizing soft } \\
\text { tissue infections }\end{array}$ & $1969-1995$ & 33 cases & Case review & $N=26$ & C \\
\hline \multicolumn{9}{|l|}{ PEM } \\
\hline Layton 2004a [58] & UK & Celecoxib & Not specified & 2000 & 17,458 patients & $\begin{array}{l}\text { IDs (event } \\
\text { incidence } \\
\text { densitites) }\end{array}$ & $\begin{array}{l}\text { Dyspepsia }=25.4 \\
\text { Abdominal pain }=10.6\end{array}$ & $A+B$ \\
\hline Layton 2003b [59] & UK & $\begin{array}{l}\text { Celecoxib } \\
\text { Meloxicam }\end{array}$ & Not specified & $1996-1997$ & 34,355 patients & PRR & $\begin{array}{l}\text { Different categories } \\
\text { reported }\end{array}$ & A \\
\hline Layton 2003 c [60] & UK & Rofecoxib & Not specified & 2000 & 15,268 patients & $\begin{array}{l}\text { Event rate pr. } 1000 \\
\text { patient months } \\
\text { exposure }\end{array}$ & $\begin{array}{l}76 \text { upper GI bleedings and } \\
101 \text { thromboembolic } \\
\text { events }\end{array}$ & $A+B$ \\
\hline Layton 2004d [6I] & UK & Rofecoxib & $\begin{array}{l}\text { Exacerbation of } \\
\text { colitis }\end{array}$ & 1999 & 15,268 patients & IRR & $5.8 ; 2.7-11.3$ & A \\
\hline Kasliwal 2005 [62] & UK & $\begin{array}{l}\text { coX-2 } \\
\text { inhibitors }\end{array}$ & $\begin{array}{l}\text { Gastrointestinal + } \\
\text { thromboembolic } \\
\text { events }\end{array}$ & $1999-2000$ & 32,726 patients & PRR & $\begin{array}{l}\text { GI: I.2I; } 1.09-1.36 \text {. } \\
\text { Thromboembolic: I.04; } \\
0.50-2.17 \text {. }\end{array}$ & $A+B$ \\
\hline Layton 2003e [63] & UK & $\begin{array}{l}\text { Rofecoxib } \\
\text { Meloxicam }\end{array}$ & $\begin{array}{l}\text { Thromboembolic } \\
\text { events }\end{array}$ & $1996-1997$ & 34,355 patients & PRR & $1.68 ; 1.15-2.46$ & $A$ \\
\hline Layton $2003 \mathrm{f}$ [64] & UK & $\begin{array}{l}\text { Rofecoxib } \\
\text { Meloxicam }\end{array}$ & Upper Gl events & $1996-1997$ & 34,355 patients & IR & $0.71 ; 0.65-0.79$ & A \\
\hline Layton 2006g [65] & UK & $\begin{array}{l}\text { COX-2 } \\
\text { inhibitors }\end{array}$ & $\begin{array}{l}\text { Serious skin } \\
\text { reactions }\end{array}$ & 1999-2000/ & 52,644 patients & $\begin{array}{l}\mathrm{IR} / \mathrm{I} 000 \text { patient- } \\
\text { months }\end{array}$ & IR: 0.019 & B \\
\hline Layton 2003h [66] & UK & $\begin{array}{l}\text { Celecoxib } \\
\text { Meloxicam }\end{array}$ & $\begin{array}{l}\text { Gastrointestinal } \\
\text { events }\end{array}$ & $1996-1997$ & 36,545 patients & PRR & $0.77 ; 0.69-0.85$ & A \\
\hline \multicolumn{9}{|l|}{ Case series } \\
\hline Onder 2004 [67] & - & NSAIDs & Psychiatric ADRs & $1965-2003$ & $\begin{array}{l}27 \text { reports with } \\
\text { data on } 453 \text { cases }\end{array}$ & Risk factors & $\begin{array}{l}\text { Age, psychiatric } \\
\text { disorders, parturients }\end{array}$ & B \\
\hline Fraunfelder 2006 [68] & - & NSAIDs & Ocular ADRs & - & 569 cases & Reported ADRs & $\begin{array}{l}\text { Blurred vision, } \\
\text { conjunctivitis, visual } \\
\text { hallucinations }\end{array}$ & B \\
\hline Zimer 2007 [69] & $\mathrm{DE}$ & Valdecoxib & $\begin{array}{l}\text { Cutaneous adverse } \\
\text { reactions }\end{array}$ & $2002-2005$ & 5 cases & Case review & $\begin{array}{l}\text { Erythematous, facial } \\
\text { edema, dyspnea }\end{array}$ & B \\
\hline \multicolumn{9}{|l|}{ Case reports } \\
\hline Hunter 1999 [70] & - & Bromfenac & Hepatic Failure & - & I case & $\begin{array}{l}\text { Causality } \\
\text { assessment }\end{array}$ & Related & B \\
\hline ADRAC I998 [7I] & - & $\begin{array}{l}\text { Diclofenac } \\
\text { Indomethacin } \\
\text { Mefenamic } \\
\text { acid }\end{array}$ & $\begin{array}{l}\text { Closure of fetal } \\
\text { ductus arterious }\end{array}$ & - & 3 cases & Case review & Case review & C \\
\hline
\end{tabular}


Table 5: Studies of the occurrence of ADRs related to SSRI use

\begin{tabular}{|c|c|c|c|c|c|c|c|c|}
\hline Reference & Setting & Medicines & ADRs & $\begin{array}{l}\text { Sampling } \\
\text { period }\end{array}$ & Sample size & $\begin{array}{l}\text { Outcome } \\
\text { measures }\end{array}$ & $\begin{array}{l}\text { Results } \\
(95 \% \mathrm{CI})\end{array}$ & $\begin{array}{l}\text { Type of } \\
\text { ADRs }\end{array}$ \\
\hline \multicolumn{9}{|l|}{$\begin{array}{l}\text { Case-control } \\
\text { studies }\end{array}$} \\
\hline Schillevoort 2002 [72] & NL & SSRIs & $\begin{array}{l}\text { Extrapyramidal } \\
\text { Syndromes (EPS) }\end{array}$ & $1985-1999$ & $\begin{array}{l}4 \text { I cases/I,264 } \\
\text { controls }\end{array}$ & OR & $2.2 ; 1.2-3.9$ & A \\
\hline Movig 2002 [73] & NL & SSRIs & Hyponatraemia & $1990-1998$ & $\begin{array}{l}203 \text { cases } / 608 \\
\text { controls }\end{array}$ & OR & $3.96 ; 1.33-11.83$ & $A$ \\
\hline \multicolumn{9}{|l|}{ Cohort study } \\
\hline Bell 2006 [74] & US & Fluoxetine & $\begin{array}{l}\text { Testosterone } \\
\text { levels }\end{array}$ & - & 14 patients & $\begin{array}{l}\text { Testosterone } \\
\text { level }\end{array}$ & No changes & B \\
\hline \multicolumn{9}{|l|}{$\begin{array}{l}\text { National ADR } \\
\text { databases }\end{array}$} \\
\hline Trenque 2002 [75] & FR & SSRIs & $\begin{array}{l}\text { Withdrawal } \\
\text { syndrome }\end{array}$ & $<2000$ & $\begin{array}{l}60 \text { cases } / 166,327 \\
\text { non cases }\end{array}$ & OR & $5.05,3.8 \mathrm{I}-6.68$ & $A$ \\
\hline Gony 2003 76] & $\mathrm{FR}$ & SSRIs & $\begin{array}{l}\text { Extrapyramidal } \\
\text { Symptoms }\end{array}$ & $1995-2000$ & 9 cases & OR & $2.18 ; 0.47-11.35$ & $A$ \\
\hline Hedenmalm 2006 [77] & SE & SSRIs & Alopecia & $<2004$ & 27 cases & IC & $\begin{array}{l}\text { Sertraline = } 1.63 \text {, } \\
0.85-2.41 \\
\text { Citalopram = } 1.22 \text {, } \\
0.97-1.47\end{array}$ & B \\
\hline Goldstein 1997 [78] & - & Fluoxetine & $\begin{array}{l}\text { First-trimester } \\
\text { exposure on } \\
\text { newborns }\end{array}$ & $<1996$ & 796 cases & Rate \% & 5.0 & C \\
\hline Spigset 2003 [79] & SE & Nefazodone & Hepatic injury & $<2002$ & $\begin{array}{l}27,542 \text { cases/ } \\
2830764 \text { non } \\
\text { cases }\end{array}$ & IC & $0.42,0.12-0.72$ & B \\
\hline Khan 2003 [80] & US & SSRIs & Suicide & $1985-2000$ & $\begin{array}{l}77 \text { cases } / 48,277 \\
\text { non cases }\end{array}$ & Suicide rate & $0.59,0.31-0.87$ & A \\
\hline Egberts I997 [8I] & NL & SSRIs & $\begin{array}{l}\text { Non-puerperal } \\
\text { lactation }\end{array}$ & $1986-1996$ & $\begin{array}{l}38 \text { cases/14,439 } \\
\text { non cases }\end{array}$ & OR & $2.7 ; 6.4-25.4$ & $A$ \\
\hline Kvande 200I [82] & NO & SSRIs & Pancreatitis & $<2000$ & 160 cases & No. of cases & 160 cases & B \\
\hline Stahl I997 [83] & SE & SSRIs & $\begin{array}{l}\text { Withdrawal } \\
\text { reactions }\end{array}$ & $<1995$ & 49,393 cases & $\begin{array}{l}\text { Number of } \\
\text { reports/106/ } \\
\text { DDD }\end{array}$ & $\begin{array}{l}\text { Paroxetine }=1.9 \\
\text { Sertraline }=2.1 \\
\text { Fluoxetine }=0.48\end{array}$ & $A$ \\
\hline Spigset 1999 [84] & SE & SSRIs & Not specified & $1965-1997$ & 1202 cases & ADRs & $\begin{array}{l}\text { Different categories } \\
\text { reported }\end{array}$ & $A+B$ \\
\hline Sanz 2005 [85] & SE & SSRIs & $\begin{array}{l}\text { Neonatal } \\
\text { withdrawal } \\
\text { syndrome }\end{array}$ & $1968-2002$ & 102 cases & IC & $\begin{array}{l}\text { Paroxetine }=4.07 \\
\text { Sertraline }=1.20 \\
\text { Citalopram }=1.92 \\
\text { Fluoxetin }=1.07\end{array}$ & C \\
\hline \multicolumn{9}{|l|}{ PEM } \\
\hline Price 1996 [86] & UK & SSRIs & $\begin{array}{l}\text { Withdrawal } \\
\text { reactions }\end{array}$ & $1987-1992$ & 50,150 patients & $\begin{array}{l}\text { Reports/ } \\
1000 \\
\text { prescriptions }\end{array}$ & $\begin{array}{l}\text { Paroxetine }=0.3 \\
\text { Sertraline }=0.03 \\
\text { Fluvoxamine }=0.03 \\
\text { Fluoxetine }=0.002\end{array}$ & $A$ \\
\hline Layton 2001 [87] & UK & SSRIs & Abnormal bleeding & $1986-1998$ & 135,754 patients & PRR & $\begin{array}{l}\text { Day } 1-30=1.38 \\
\text { Month } 2-6=1.17\end{array}$ & A \\
\hline
\end{tabular}


Table 5: Studies of the occurrence of ADRs related to SSRI use (Continued)

\begin{tabular}{|c|c|c|c|c|c|c|c|c|}
\hline Edwards 1994 [88] & UK & Fluvoxamine & All & $1987-1988$ & I0,40| patients & Incidences & & A \\
\hline MacKay 1997 [89] & UK & SSRIs & All & |988-199| & 56,145 patients & & $\begin{array}{l}\text { Nausea, vomiting, } \\
\text { withdrawal } \\
\text { symptoms }\end{array}$ & \\
\hline \multicolumn{9}{|l|}{ Case series } \\
\hline de Abajo 2006 [90] & - & $\begin{array}{l}\text { SSRIs } \\
\text { Venlafaxine }\end{array}$ & Bleeding Disorders & $1988-2003$ & $\begin{array}{l}\text { I,65I cases/ } \\
\text { I0,000 controls }\end{array}$ & PRR & $3.0,2.1-4.4$ & A \\
\hline Gram 1999 [91] & DK & SSRIs & $\begin{array}{l}\text { Bleeding } \\
\text { Thrombocytopenia }\end{array}$ & - & 8 cases & - & Case review & $A+B$ \\
\hline \multicolumn{9}{|l|}{ Case report } \\
\hline Demers 200I [92] & - & Fluvoxamine & $\begin{array}{l}\text { Serotonin } \\
\text { syndrome }\end{array}$ & - & I case & - & Case review & A \\
\hline
\end{tabular}

\section{NSAIDs}

Studies explored the risk of gastrointestinal [37-44,50] and dermatological ADRs as well as the development of liver and kidney toxicity which are well known ADRs associated with NSAIDs and their pharmacological characteristics $[38,39,48,51,53,54,57,65,69,70]$. The studies were generated after the launch of COX-2 inhibitors in the mid1990s. A case-control study documented increased risk of developing dermatological ADRs of the type Steven-Johnson Syndrome and toxic epidermal necrolysis as did spontaneously reported ADRs [38,48]. A case-control study documented hepatic injury related to the use of NSAIDs, as did spontaneously reported ADRs, while renal injury and hypertension was documented in spontaneous reports and thromboembolic events in a PEM study $[39,45,47,51,53,54,63,70]$. With the exception of case reports, the approaches used did not produce information about ADRs that had not been reported previously.

\section{SSRIS}

Studies explored the risk of extrapyramidal symptoms, withdrawal syndromes and serotonin syndrome with the use of SSRIs, other ADRs investigated were: changes in testosterone and natrium level, alopecia, liver injury and bleeding. ADRs reported only via spontaneous reports are first-trimester exposure on newborns and neonatal withdrawal syndrome, hepatic injury and pancreatitis, suicide, non-puerperal lactation and serotonin syndrome [72$81,79,83,85,86,90-92]$. With the exception of case reports, the approaches used did not detect new ADR signals that had not been reported previously [90-92].

\section{Information about ADRs reported across approaches Analytical}

The approaches produced information about ADR risks compared to placebo or similar drugs as either odds ratios (OR), proportional reporting ratio (PRR) estimates, incidences (IC) and frequencies of ADRs. These parameters are built into the design and based on previous information or hypothesis. The studies were conducted on various patient populations, various medicines within the individual sub-groups, and different types of ADRs, different outcome measures, data sources and time periods. The purpose of the approaches made it possible to adjust the ADR estimate for known confounders and risk factors.

\section{Observational}

The approaches produced information about ADRs as estimates (OR, PRR, IC) or as single observations compared to placebo/similar medicines. Case reporting was the only approach that contributed new information about new ADRs in all three therapeutic cases.

\section{Discussion}

This review has several main findings:

First, analytical approaches ranging higher in the evidence hierarchy provided information about risks of already known or expected ADRs, while information about new and unknown ADRs was detected by case reports only, which range at the lowest level in the evidence hierarchy. Second, the studies primarily dealt with analyses of ADRs of type A and B, and only a few studies analysed type $\mathrm{C}$ and D. Third, similar approaches, both analytical and observational, were applied to all therapeutic cases. Fourth, the ADR cases provided similar results with regard to detecting new ADRs despite their connection to different time periods and organs attacked.

\section{Methodological quality and capability of approaches}

There is a general lack of standards in the field of ADRs, particularly because many ADRs are not detected until after marketing and the studies are based on selected patient groups, which makes it difficult to generalise the results to other patient groups. As previously argued in the literature, testing specific hypotheses in the analytical 
Table 6: Number of studies categorised by number, design and time of publication

\begin{tabular}{|c|c|c|c|c|c|c|c|c|c|c|c|c|c|c|c|c|}
\hline $\begin{array}{l}\text { Year of } \\
\text { publication }\end{array}$ & $<1990$ & 1991 & 1992 & 1993 & 1994 & 1995 & 1996 & 1997 & 1998 & 1999 & 2000 & 2001 & 2002 & 2003 & 2004 & 2005- \\
\hline \multicolumn{17}{|l|}{ Study design } \\
\hline \multicolumn{17}{|l|}{ Antibiotics } \\
\hline $\begin{array}{l}\text { Case control } \\
\text { studies }\end{array}$ & & & & & & & & & & 1 & & & & & & I \\
\hline Cohort studies & & 1 & & 3 & & & & & & & & & & & & I \\
\hline $\begin{array}{l}\text { National ADR } \\
\text { databases }\end{array}$ & & & & & & & 1 & & & & 2 & & & 1 & & 2 \\
\hline PEM* & & & & & 1 & & & & & & & I & & & & \\
\hline Case series & & & & & & & & & & & & & 1 & & & I \\
\hline Case reports & & & 1 & & & & 1 & 1 & & & 1 & & & 1 & & \\
\hline \multicolumn{17}{|l|}{ NSAIDs } \\
\hline $\begin{array}{l}\text { Case control } \\
\text { studies }\end{array}$ & & & & & & & & & & & I & 1 & & 1 & I & \\
\hline Cohort studies & & & & & & & & & & & I & & & & 3 & \\
\hline $\begin{array}{l}\text { National ADR } \\
\text { databases }\end{array}$ & & & & & & & & 1 & I & I & I & I & I & 1 & 4 & 3 \\
\hline PEM* $*$ & & & & & & & & & & & & & & 5 & 2 & 2 \\
\hline Case series & & & & & & & & & & & & & & & 1 & 2 \\
\hline Case reports & & & & & & & & & I & 1 & & & & & & \\
\hline \multicolumn{17}{|l|}{ SSRIs } \\
\hline $\begin{array}{l}\text { Case control } \\
\text { studies }\end{array}$ & & & & & & & & & & & & & 2 & & & \\
\hline Cohort studies & & & & & & & & & & & & & & & & I \\
\hline $\begin{array}{l}\text { National ADR } \\
\text { databases }\end{array}$ & & & & & & & & 3 & & I & & I & 1 & 3 & & 2 \\
\hline PEM* & & & & & I & & 1 & I & & & & I & & & & \\
\hline Case series & & & & & & & & & & 1 & & & & & & I \\
\hline Case reports & & & & & & & & & & & & I & & & & \\
\hline
\end{tabular}

*Prescription Event Monitoring Studies

approaches makes it difficult to capture information about new and unknown ADRs [13]. Despite the fact that these types of studies rank high in the evidence hierarchy, the weaker design of the observational studies makes them more suitable for discovering previously undetected ADRs. Healthcare professionals have conventionally considered cohort and case-control studies to be well suited for post-marketing surveillance of ADRs, despite their lack of randomisation and lower position in the evidence hierarchy, level 4 and 5 respectively [14]. These studies primarily detected/analysed ADRs of type A and B and less frequently type $\mathrm{C}$ and $\mathrm{D}$ [14]. Thus, the approaches are not designed and therefore are not suitable for predicting new information about other ADRs that have not previously been detected or ADRs of the type C or D [14]. Case reports have provided data about patients, suspected ADRs, medicines involved and so on, but this information is often anecdotal in nature and collected retrospectively. However, it is interesting that despite their low rank in the evidence hierarchy, these reports provide new information about rare and previous undetected ADRs. Case reports may serve as whistleblowers, thereby initiating larger systematic analyses of patient populations or registering data to quantify the risk. A large majority of spontaneously reported ADRs are stored in databases hosted by regulatory agencies. Information about these observations is typically only released to the public in the form of press releases, insertions in product information or messages in national bulletins. If all these signals were published in the scientific literature or made public on the web pages of regulatory agencies, the number of spontaneous reports/case series would probably have been larger and added to the relative dominance of this design $[93,94]$. The results confirm that spontaneous post-marketing 
reporting of ADRs is of great importance and that regulatory agencies must continue to encourage spontaneous reporting of ADRs [93,94].

\section{Alternative signal detection approaches}

New ADR signals are often documented by only a small number of case reports, and systematic inclusion of data mining procedures in assessment of new ADR signals would probably contribute to earlier detection and quantification of serious ADR signals $[95,96]$. However, data mining was not applied in the three therapeutic cases studied here. Examples of data mining are cumulative techniques, time scans and Poisson methods, proportional reporting ratios (PRRs) and Bayesian data mining [97]. These methods assess how much the observed reporting frequency of a given drug-event combination deviates from that expected, given statistical independence between drug and event. Methodological and practical experiences with data mining in signal detection are limited $[97,98]$.

\section{Strengths and limitations of the study}

The objective of this review was to analyse which information signal detection approaches have produced about new ADRs in selected and published therapeutic cases, rather than to perform a systematic review of the entire body of ADR literature covering all therapeutic groups. The choice of widely different therapeutic cases and the similar results obtained across therapeutic cases make us believe that the results qualitatively reflect the general, published experience on ADRs based on signal detection approaches. Findings across therapeutic cases were similar with respect to methodological approaches and time of publication, despite the fact that ADRs differed in nature and affected different organs. Although antibiotics have been marketed since the 1940s, it was not possible to search for literature before the mid-1960s due to the limitations of current databases. Lack of consistency in reporting ADRs, different methodologies used in the studies and their impact on the results are difficult to evaluate in this review.

\section{Conclusion}

Although the medicines were launched in different decades, approaches to the ADR studies were similar for all three therapeutic cases: antibiotics, NSAIDs and SSRIs. Descriptive as well as analytical designs were applied. Despite the fact that the analytical studies rank higher in the evidence hierarchy, only the descriptive case reports/ spontaneous reports provided information about new and previously undetected ADRs. This review underscores the importance of systems for spontaneous reporting of ADRs. Therefore, spontaneous reporting should be encouraged further and the information in ADR databases should continuously be subjected to systematic analysis.

\section{Competing interests}

The authors declare that they have no competing interests.

\section{Authors' contributions}

LA and EHH designed the study, analysed the data and wrote the various drafts of the manuscript. LA did the sampling and literature search. Both authors read and approved the final version of the manuscript.

\section{Acknowledgements}

We thank The Danish Medicines Agency and the Hørslev Foundation for their financial support of the study.

\section{References}

I. Inman WHW, Vessey MP, Westerholm B, Engelund A: Thromboembolic disease and the steroidal content of oral contraceptives. A report to the Committee on Safety of Drugs. BMJ 1970, 25:203-9.

2. Dukes MNG: The effects of drug regulation. Lancaster: MTP Press Limited; 1985.

3. Horton R: Vioxx, the implosion of Merck, and the after shocks at the FDA. Lancet 2004, 364:1995-6.

4. Tscholl D, Langer F, Wendler O, Wilkens H, Georg T, Schäfers HJ: Pulmonary thromboendarterectomy- risk factors for early survival and hemodynamic improvement. Eur J Cardiothorac Surg 200I, 19:77I-6.

5. Stefan H, Bernatik J, Knorr J: Visual field defects due to antiepileptic drugs. Nervenartz 1999, 70:552-5.

6. Watkins P: COMT inhibitors and liver toxicity. Neurology 2000 , 55:S5I-2.

7. Aagaard L, Soendergaard B, Andersen E, Kampmann JP, Hansen EH: Creating knowledge about adverse drug reactions: a critical analysis of the Danish reporting system from 1968 to 2005. Soc Sci Med 2007, 65(6): 1296-309.

8. Hansen EH: Technology assessment of pharmaceuticals - the necessity of user perspective. Cah Sociol Demogr Med 1990, 30:313-27.

9. Hansen EH: Technology assessment in a user perspective experiences with drug technology. Int J Technol Assess Health Care 1992, 8: 150-65.

10. Dukes MNG: The law and ethics of the pharmaceutical industry Amsterdam, Elsevier; 2006.

II. Loke YK, Derry S, Aronson JK: A comparison of three different sources of data in assessing the frequencies of adverse reactions to amiodarone. BrJ Clin Pharmacol 2004, 57(5):616-62I.

12. Loke YK, Price D, Herxheimer A: Systematic reviews of adverse effects: framework for a structured approach. BMC Medical Research Methodology 2007, 7:32.

13. Strom BL: Pharmacoepidemiology West Sussex, John Wiley \& Sons Ltd; 2005.

14. Rawlins MD, Thompson JW: Pathogenesis of adverse drug reactions. Textbook of Adverse. Drug Reactions 1977:10-31.

15. Czeizel AE, Rockenbauer M, Sørensen HT, Olsen J: A populationbased case-control teratologic study of oral erythromycin treatment during pregnancy. Reprod Toxicol 1999, 1 3:531-6.

16. Seeger JD, West WA, Fife D, Noel GJ, Johnson LN, Walker AM: Achilles tendon rupture and its association with fluoroquinolone antibiotics and other potential risk factors in a managed care population. Pharmacoepidemiol Drug Saf 2006, 15:784-92.

17. Chyský V, Kapila K, Hullmann R, Arcieri G, Schacht P, Echols R: Safety of ciprofloxacin in children: worldwide clinical experience based on compassionate use. Emphasis on joint evaluation. Infection |99|, 19:289-96.

18. Derby LE, Jick H, Henry DA, Dean AD: Cholestatic hepatitis associated with flucloxacillin. Med J Aust 1993, 158:596-00.

19. Jick H, Derby LE, Dean AD, Henry DA: Flucloxacillin and cholestatic hepatitis. Med J Aust 1994, 18:160-525.

20. Derby LE, Jick H, Henry DA, Dean AD: Erythromycin-associated cholestatic hepatitis. Med J Aust 1993, 158:600-2. 
21. Heymann AD, Chodick G, Kramer E, Green M, Shalev V: Pemphigus variant associated with penicillin use: a case-cohort study of 363 patients from Israel. Arch Dermatol 2007, I 43:704-7.

22. Clark DW, Layton D, Wilton LV, Pearce GL, Shakir SA: Profiles of hepatic and dysrhythmic cardiovascular events following use of fluoroquinolone antibacterials: experience from large cohorts from the Drug Safety Research Unit PrescriptionEvent Monitoring database. Drug Saf 200 I, 24:I I 43-54.

23. Inmann W, Pearce G, Wilton L: Safety of fluconazole in the treatment of vaginal candidiasis. A prescription-event monitoring study, with special reference to the outcome of pregnancy. Eur J Clin Pharmacol 1994, 46: I I5-8.

24. Sachs B, Riegel S, Seebeck J, Beier R, Schichler D, Barger A, Merk HF, Erdmann S: Fluoroquinolone-associated anaphylaxis in spontaneous adverse drug reaction reports in Germany: differences in reporting rates between individual fluoroquinolones and occurrence after first-ever use. Drug Saf 2006, 29:1087-00.

25. Fleisch F, Hartmann K, Kuhn M: Fluoroquinolone-induced tendinopathy: also occurring with levofloxacin. Infection 2000, 28:256-7.

26. Leone R, Venegoni M, Motola D, Moretti U, Piazzetta V, Cocci A, Resi D, Mozzo F, Velo G, Burzilleri L, Montanaro N, Conforti A: Adverse drug reactions related to the use of fluoroquinolone antimicrobials: an analysis of spontaneous reports and fluoroquinolone consumption data from three Italian regions. Drug Saf 2003, 26:109-20.

27. Pierfitte C, Royer RJ, Moore N, Bégaud B: The link between sunshine and phototoxicity of sparfloxacin. BrJ Clin Pharmacol 2000 49:609-12.

28. Frothingham R: Glucose homeostasis abnormalities associated with use of gatifloxacin. Clin Infect Dis 2005, 4I:I269-76.

29. Hedenmalm K, Spigset O: Peripheral sensory disturbances related to treatment with fluoroquinolones. J Antimicrob Chemother 1996, 37:831-7.

30. Abouesh A, Stone C, Hobbs WR: Antimicrobial-induced mania (antibiomania): a review of spontaneous reports. J Clin Psychopharmacol 2002, 22:7I-8I.

3I. Smith K, Leyden JJ: Safety of doxycycline and minocycline: a systematic review. Clin Ther 2005, 27:1329-42.

32. Hällgren J, Tengvall-Linder M, Persson M, Wahlgren CF: StevensJohnson syndrome associated with ciprofloxacin: a review of adverse cutaneous events reported in Sweden as associated with this drug. J Am Acad Dermatol 2003, 49:S267-9.

33. Warner A: Clarithromycin-a precipitant for acute psychotic stress. Psychosomatics 2000, 41:539.

34. ADRAC: Drug-induced cholestatic hepatitis from common antibiotics. MJA 1992, 157:531.

35. Greco S, Mazzaglia G, Caputi AP, Pagliaro L: Glossitis, stomatitis, and black tongue with lansoprazole plus clarithromycin and other antibiotics. Ann Pharmacother 1997, 3 1:1548.

36. Björnsson E, Lindberg J, Olsson R: Liver reactions to oral lowdose tetracyclines. Scand J Gastroenterol 1997, 32:390-5.

37. Hernández-Díaz S, Rodríguez LA: Steroids and risk of upper gastrointestinal complications. Am J Epidemiol 200I, 153:1089-93.

38. Mockenhaupt M, Kelly JP, Kaufman D, Stern RS, SCAR Study Group: The risk of Stevens-Johnson syndrome and toxic epidermal necrolysis associated with nonsteroidal antiinflammatory drugs: a multinational perspective. I Rheumatol 2003, 30:2234-40.

39. Lacroix I, Lapeyre-Mestre M, Bagheri H, Pathak A, Montastruc JL: Nonsteroidal anti-inflammatory drug-induced liver injury: a case-control study in primary care. Fundam Clin Pharmacol 2004 I8:20I-6.

40. Lipworth L, Friis S, Blot WJ, McLaughlin JK, Mellemkjaer L, Johnsen SP, Nørgaard B, Olsen JH: A population-based cohort study of mortality among users of ibuprofen in Denmark. Am J Ther 2004, II:156-63

41. Ashworth NL, Peloso PM, Muhajarine N, Stang M: A population based historical cohort study of the mortality associated with nabumetone, Arthrotec, diclofenac, and naproxen. Rheumatol 2004, $31: 951-6$.

42. Morant SV, Pettitt D, MacDonald TM, Burke TA, Goldstein JL: Application of a propensity score to adjust for channelling bias with NSAIDs. Pharmacoepidemiol Drug Saf 2004, 13:345-53.

43. Martin RM, Biswas $P$, Mann RD: The incidence of adverse events and risk factors for upper gastrointestinal disorders associ- ated with meloxicam use amongst 19,087 patients in general practice in England: cohort study. Br J Clin Pharmacol 2000, 50:35-42.

44. Lugardon S, Lapeyre-Mestre M, Montastruc JL: Upper gastrointestinal adverse drug reactions and cyclo-oxygenase- 2 inhibitors (celecoxib and rofecoxib): a case/non-case study from the French Pharmacovigilance Database. Eur J Clin Pharmacol 2004, 60:673-7.

45. Durrieu G, Olivier P, Montastruc JL: COX-2 inhibitors and arterial hypertension: an analysis of spontaneous case reports in the Pharmacovigilance database. Eur J Clin Pharmacol 2005, 6I:6II-4.

46. Clinard F, Sgro C, Bardou M, Hillon P, Dumas M, Kreft-Jais C, Escousse A, Bonithon-Kopp C: Association between concomitant use of several systemic NSAIDs and an excess risk of adverse drug reaction. A case/non-case study from the French Pharmacovigilance system database. Eur J Clin Pharmacol 2004, 60:279-83.

47. Brinker A, Goldkind L, Bonnel R, Beitz J: Spontaneous reports of hypertension leading to hospitalisation in association with rofecoxib, celecoxib, nabumetone and oxaprozin. Drugs Aging 2004, $21: 479-84$

48. La Grenade L, Lee L, Weaver J, Bonnel R, Karwoski C, Governale L, Brinker A: Comparison of reporting of Stevens-Johnson syndrome and toxic epidermal necrolysis in association with selective COX-2 inhibitors. Drug Saf 2005, 28:917-24.

49. Polimeni G, Salvo F, Cutroneo P, Morreale I, Patrizio Caputi A: Adverse reactions induced by NSAIDs and antibacterials: analysis of spontaneous reports from the Sicilian regional database. Drug Saf 2006, 29:449-59.

50. Conforti A, Leone R, Moretti U, Mozzo F, Velo G: Adverse drug reactions related to the use of NSAIDs with a focus on nimesulide: results of spontaneous reporting from a Northern Italian area. Drug Saf 200I, 24: I08I-90.

5I. Ahmad SR, Kortepeter C, Brinker A, Chen M, Beitz J: Renal failure associated with the use of celecoxib and rofecoxib. Drug Saf 2002, 25:537-44

52. van Puijenbroek EP, Egberts AC, Heerdink ER, Leufkens HG: Detecting drug-drug interactions using a database for spontaneous adverse drug reactions: an example with diuretics and nonsteroidal anti-inflammatory drugs. Eur J Clin Pharmacol 2000, 56:733-8.

53. Lapeyre-Mestre M, de Castro AM, Bareille MP, Del Pozo JG, Requejo AA, Arias LM, Montastruc JL, Carvajal A: Non-steroidal antiinflammatory drug-related hepatic damage in France and Spain: analysis from national spontaneous reporting systems. Fundam Clin Pharmacol 2006, 20:39I-5.

54. Leone R, Conforti A, Ghiotto E, Moretti U, Valvo E, Velo GP: Nimesulide and renal impairment. Eur J Clin Pharmacol 1999, 55: $151-4$.

55. Brown EG, Waller PC, Sallie BA: Tiaprofenic acid and severe cystitis. Postgrad Med J 1998, 74:443-4.

56. Verrico MM, Weber RJ, McKaveney TP, Ansani NT, Towers AL: Adverse Drug Events Involving COX-2 Inhibitors. Ann Pharmacother 2003, 37:1203-13.

57. Kahn LH, Styrt BA: Necrotizing soft tissue infections reported with nonsteroidal antiinflammatory drugs. Ann Pharmacother 1997, 31 : 1034-9.

58. Layton D, Wilton LV, Shakir SA: Safety profile of celecoxib as used in general practice in England: results of a prescriptionevent monitoring study. Eur I Clin Pharmacol 2004, 60:489-0I.

59. Layton D, Hughes K, Harris S, Shakir SA: Comparison of the incidence rates of selected gastrointestinal events reported for patients prescribed celecoxib and meloxicam in general practice in England using prescription-event monitoring (PEM) data. Rheumatology (Oxford) 2003, 42: |332-4I.

60. Layton D, Riley J, Wilton LV, Shakir SA: Safety profile of rofecoxib as used in general practice in England: results of a prescription-event monitoring study. Br J Clin Pharmacol 2003, 55: I 66-74.

61. Layton D, Heeley E, Shakir SA: Identification and evaluation of a possible signal of exacerbation of colitis during rofecoxib treatment, using Prescription-Event Monitoring data. J Clin Pharm Ther 2004, 29: 17I-8I.

62. Kasliwal R, Layton D, Harris S, Wilton L, Shakir SA: A comparison of reported gastrointestinal and thromboembolic events 
between rofecoxib and celecoxib using observational data. Drug Saf 2005, 28:803-16.

63. Layton D, Heeley E, Hughes K, Shakir SA: Comparison of the incidence rates of thromboembolic events reported for patients prescribed rofecoxib and Meloxicam in general practice in England using Prescription-event monitoring (PEM) data. Rheumatology 2003, 42: I342-53.

64. Layton D, Heeley E, Hughes K, SAW Shakir: Comparison of the incidence rates of selected gastrointestinal events reported for patients prescribed rofecoxib and meloxicam in general practice in England using prescription-event monitoring data. Rheumatology 2003, 42:622-31.

65. Layton D, Marshall V, Boshier A, Friedmann P, Shakir SA: Serious Skin Reactions and Selective COX-2 Inhibitors. A Case Series from Prescription-Event Monitoring In England. Drug Saf 2006, 29:687-96.

66. Layton D, Hughes K, Harris S, Shakir SA: Comparison of the incidence rates of thromboembolic events reported for patients prescribed celecoxib and meloxicam in general practice in England using Prescription-Event Monitoring (PEM) data. Rheumatology 2003, 42: 1354-64.

67. Onder G, Pellicciotti F, Gambassi G, Bernabei R: NSAID-Related Psychiatric Adverse Events. Who is at risk? Drugs 2004, 64:2619-27.

68. Fraunfelder FW, Solomon J, Mechelas TJ: Ocular Adverse Effects Associated With Cyclooxygenase-2 Inhibitors. Arch Ophthal$\mathrm{mol}$ 2006, I 24:277-79.

69. Ziemer M, Wiesend CL, Vetter R, Weiss J, Blaschke S, Norgauer J, Mockenhaupt M: Cutaneous Adverse Reactions to Valdecoxib Distinct From Stevens-Johnson Syndrome and Toxic Epidermal Necrolysis. Arch Dermatol 2007, I 43:7 I I-I6.

70. Hunter EB, Johnston PE, Tanner G, Pinson CW, Awad JA: Bromfenac (Duract)- Associated Hepatic Failure Requiring Liver Transplantation. AJG 1999, 94:2299-0I.

71. ADRAC: Premature closure of the fetal ductus arteriosus after maternal use of non-steroidal anti-inflammatory drugs. MJA 1998, 169:270-7I.

72. Schillevoort I, van Puijenbroek EP, de Boer A, Ross RA, Jansen PA, Leufkens HG: Extrapyramidal syndromes associated with selective serotonin reuptake inhibitors: a case-control study using spontaneous reports. Int Clin Psychopharmacol 2002, I 7:75-9.

73. Movig KLL, Leufkens HGM, Lenderink AW, Eberts ACG: Serotonergic antidepressants associated with an increased risk for hyponatraemia in the elderly. Eur J Clin Pharmacol 2002, 58: $143-48$.

74. Bell S, Shipman M, Bystritsky A, Haifley T: Fluoxetine Treatment and Testosterone Levels. Annals of Clinical Psychiatry 2006 18:19-22.

75. Trenque T, Piednoir D, Frances C, Millart H, Germain ML: Reports of withdrawal syndrome with the use of SSRIs: a case/noncase study in the French Pharmacovigilance database. Pharmacoepidemiol Drug Saf 2002, I I:28I-3.

76. Gony M, Lapeyre-Mestre M, Montastruc JL: Risk of serious extrapyramidal symptoms in patients with Parkinson's disease receiving antidepressant drugs: a pharmacoepidemiologic study comparing serotonin reuptake inhibitors and other antidepressant drugs. Clin Neuropharmacol 2003, 26: I 42-5.

77. Hedenmalm K, Sundström A, Spigset O: Alopecia associated with treatment with selective serotonin reuptake inhibitors (SSRIs). Pharmacoepidemiol Drug Saf 2006, 15:719-25

78. Goldstein DJ, Corbin LA, Sundell KL: Effects of First-Trimester Fluoxetine Exposure on the Newborn. Obstet Gynecol 1997 89:7I3-8.

79. Spigset $O$, Hägg $S$, Bate $A$ : Hepatic injury and pancreatitis during treatment with serotonin reuptake inhibitors: data from the World Health Organization database of adverse drug reactions. Int Clin Psychopharmacol 2003, 18:157-6I.

80. Khan A, Khan S, Kolts R, Brown WA: Suicide Rates in Clinical Trials of SSRIs, other Antidepressants and placebo: Analysis of FDA Reports. Am J Psychiatry 2003, 160:790-92.

81. Egberts ACG, Meyboom RHB, De Koning FHP, Bakker A, Leufkens HGM: Non-puerperal lactation associated with antidepressant drug use. Br J Clin Pharmacol 1997, 44:277-81.

82. Kvande KT, Madsen S: Selective serotonin reuptake inhibitors and pancreatitis. Tidsskr Nor Laegeforen 200 I, I 2 I: 177-8.
83. Stahl MMS, Lindquist M, Pettersson M, Edwards IR, Sanderson $J \mathrm{H}$ Taylor NFA, Fletcher AP, Schou JS: Withdrawal reactions with selective serotonin re-uptake inhibitors as reported to the WHO system. Eur J Clin Pharmacol 1997, 53:163-69.

84. Spigset O: Adverse Reactions of Selective Serotonin Reuptake Inhibitors. Reports from a Spontaneous Reporting System. Drug Saf 1999, 20:277-87.

85. Sanz EJ, De-las-Cuevas C, Kiuru A, Bate A, Edwards R: Selective serotonin reuptake inhibitors in pregnant women and neonatal withdrawal syndrome: a database analysis. Lancet 2005 , 365:482-87.

86. Price JS, Waller PC, Wood SM, MacKay AV: A comparison of the post-marketing safety of four selective serotonin re-uptake inhibitors including the investigation of symptoms occurring on withdrawal. Br J Clin Pharmacol 1996, 42:757-63.

87. Layton D, Clark DW, Pearce GL, Shakir SA: Is there an association between selective serotonin reuptake inhibitors and risk of abnormal bleeding? Results from a cohort study based on prescription event monitoring in England. Eur J Clin Pharmacol 200I, 57:167-76.

88. Edwards JG, Inman WH, Wilton L, Pearce G: Prescription-event monitoring of 10,401 patients treated with fluvoxamine. $\mathrm{Br} J$ Psychiatry 1994, 164:387-95.

89. MacKay FJ, Dunn NR, Wilton LV, Pearce GL, Freemantle SN, Mann RD: A comparison of fluvoxamine, fluoxetine, sertraline and paroxetine examined by observational cohort studies. Pharmacoepidemiol Drug Saf 1997, 6:235-46.

90. De Abajo FJ, Montero D, Garcia Rodriguez LA, Madurga M: Antidepressants and Risk of Upper Gastrointestinal Bleeding. Pharmacology \& Tocicology 2006, 98:304-10.

91. Gram LF: Blødning og trombocytopeni ved behandling med selektive SSRI [In Danish]. Ugeskr Laeger 1999, 16 I:2697-98.

92. Demers JC, Malone M: Serotonin Syndrome Induced by Fluvoxamine and Mirtazapine. Ann Pharmacother 200I, 35:1217-20.

93. Aagaard L, Soendergaard B, Andersen E, Kampmann JP, Hansen EH Creating knowledge about adverse drug reactions: a critical analysis of the Danish reporting system from 1968 to 2005. Soc Sci Med 2007, 65:1296-309.

94. Aagaard L, Stenver DI, Hansen EH: Structures and processes in spontaneous ADR reporting systems: a comparative study of Australia and Denmark. Pharm World Sci 2008, 30:563-70.

95. Mann RD: Drug safety alerts - a review of "current problems". Pharmacoepidemiol Drug Saf 1992, I:269-79.

96. Meyboom RHB, Gribnau FWJ, Hekster YA, de Koning GHP, Egberts ACG: Characteristics of Topics in Pharmacovigilance in the Netherlands. Clin Drug Invest 1996, I 2:207-19.

97. Hauben $M$, Zhou $X$ : Quantitative methods in pharmacovigilance: focus on signal detection. Drug Saf 2003, 26:159-86.

98. Matsushita Y, Kuroda Y, Niwa S, Sonehara S, Hamada C, Yoshimura I: Criteria Revision and Performance Comparison of Three Methods of Signal Detection Applied to the Spontaneous Reporting Database of a Pharmaceutical Manufacturer. Drug Saf 2007, 30:7I5-26.

\section{Pre-publication history}

The pre-publication history for this paper can be accessed here:

http://www.biomedcentral.com/1472-6904/9/4/prepub 